Sistematización y segmentación: dinámica histórica y expresiones actuales 



\title{
La sistematización estatal modelizadora y la segmentación en los orígenes y expansión de la escuela secundaria en la Argentina
}

Felicitas Acosta

Universidad Nacional de General Sarmiento, Argentina

facosta@campus.ungs.edu.ar

\begin{abstract}
Resumen
El trabajo parte de la hipótesis de la expansión de la escuela secundaria sobre la base de una dinámica de incorporación y expulsión simultánea en la cual el modelo institucional de origen, centrado en el colegio nacional, parece haber desempeñado un papel clave. En particular, analiza el proceso de sistematización estatal modelizadora por medio del que dicho modelo podría haber operado como institución determinante en el desarrollo de otras modalidades de enseñanza secundaria. Avanza luego sobre los efectos de la sistematización estatal modelizadora en la forma que adoptó la segmentación durante la expansión de la escuela secundaria. Utiliza fuentes primarias como decretos y reglamentos oficiales y fuentes secundarias sobre el tema.
\end{abstract}

Palabras clave: Escuela secundaria, colegio nacional, institución determinante, configuración, expansión

The State's modelling systematisation and segmentation in the origin and expansion of the secondary school in Argentina

\footnotetext{
Abstract

This article examines the hypothesis that the secondary school expansion was based on a simultaneously occurring incorporation-expulsion dynamics where the institutional model focused on the "national high schools" seems to have taken a key role. This paper specifically analyses the process of the State's modelling systematisation which might have allowed such model to operate as a defining institution in the development of other secondary school forms. The article then explores the effects of the abovementioned systematisation and discusses the form adopted by the segmentation during the secondary school expansion. Both primary sources, i.e. official decrees and regulations, and secondary sources on this subject are quoted.
} 


\section{Introducción}

A partir de hipótesis desarrolladas en estudios previos sobre la expansión de la escuela secundaria en la Argentina, este trabajo sostiene que su configuración y expansión se produjo sobre una dinámica de incorporación y expulsión simultánea, dinámica que se apoyó sobre la continuidad del modelo institucional de origen junto con el sostenimiento de una estructura única en dicha escuela (Acosta, 2008, 2012, 2015).

Propone que la forma identificada para el modelo institucional en su carácter de institución determinante (Steedman, 1992) fue la de modelización. En efecto, el Estado nacional, a través de la creación y modificación de distintos tipos de oferta destinada a la segunda enseñanza, llevó a cabo un proceso de sistematización sobre la base de una tendencia modelizadora. Dicha tendencia estaría asociada a las formas que adoptó la segmentación en el sistema educativo argentino que denominamos segmentación por diferenciación institucional.

La sistematización estatal modelizadora operó, en un comienzo, a través del modelo institucional del colegio nacional como referencia para creaciones provinciales y para escuelas particulares; luego mediante la unificación de las distintas ofertas en ciclos comunes que siguieron un proceso de generalización creciente en sus asignaturas. Todas las ofertas participaron, en mayor o menor medida y con ritmos diferentes, de este proceso, que a su vez se vio favorecido por la propia práctica de modelización entre las primeras escuelas comerciales y, sobre todo, entre las industriales. Todo ello acompañado por la unificación de reglamentos de las distintas modalidades desde 1940, consolidada hacia fines de los años ‘ 50.

Los conceptos de los que se nutre el trabajo provienen de los estudios histórico-comparativos sobre el desarrollo de los sistemas educativos y las instituciones de educación secundaria. Algunos de estos conceptos ya constituyen clásicos en la literatura sobre el tema, mientras que otros han tenido escasa circulación (Acosta, 2014).

Los sistemas educativos, de acuerdo con Mueller (1992), se configuraron sobre dos procesos: sistematización y segmentación. Estos permiten analizar la forma en que se desplegó la expansión de la educación secundaria: el primero hace referencia a la articulación de las instituciones educativas a través de certificaciones, exámenes y planes de estudio, mientras que el segundo, la segmentación, remite a la diversificación de la enseñanza secundaria y la forma en que esta tuvo lugar: creación de niveles educativos y modalidades.

Según Ringer (1979), la segmentación consistió en el desarrollo de recorridos educativos conforme el lugar a ocupar en el mercado de trabajo. La investigación educativa comprobó que esos lugares se correspondían con las posiciones sociales de origen. El autor distingue dos formas básicas de segmentación: vertical y horizontal. La creación de niveles educativos es un ejemplo de segmentación vertical, mientras que la diferenciación inter e intrainstitucional constituye ejemplos de segmentación horizontal. Cabe destacar que la distinción público/privado atraviesa las formas que adoptó la segmentación.

En los países de modernización educativa temprana, la expansión de la escuela secundaria guarda relación con la segmentación y la forma de diferenciación inter e intrainstitucional. Durante la configuración de los sistemas educativos en la última parte del siglo XIX, la diferenciación interinstitucional constituyó la primera forma de segmentación 
a través de la creación de tipos y modalidades educativas; por ejemplo bachilleratos y escuelas técnicas, comerciales o normales. El modelo de referencia fue el de los bachilleratos, de mayor valor social por sus finalidades: el acceso a la Universidad o la burocracia estatal. La lenta expansión de la educación secundaria durante la primera parte del siglo XX se hizo sobre la base de este modelo de formación generalista (Viñao, 2002). La expansión de la educación secundaria a partir de la posguerra se vincula con la acentuación de una forma más sofisticada del proceso de segmentación: la diferenciación intrainstitucional. El desarrollo de ramas paralelas, secciones, turnos, clases avanzadas, constituyen ejemplos de este tipo de diferenciación.

Nos centramos en el proceso de sistematización desde la forma en la que operó el Estado argentino en el desarrollo de la oferta de educación secundaria, proceso al cual denominamos sistematización estatal modelizadora. También consideraremos la forma de la segmentación por diferenciación interinstitucional como propia del caso de referencia. ${ }^{1}$

En cuanto a los conceptos históricos sobre las instituciones de educación secundaria, proponemos en este trabajo el de modelo institucional, que se nutre de los aportes de Peter Burke (2007) y Hilary Steedman (1992). El carácter modélico de una institución particular constituye el foco de atención. Para Burke (2007), un modelo es un constructo intelectual que simplifica la realidad a fin de destacar lo recurrente, lo general y lo típico presenta estos aspectos bajo la forma de conjuntos o rasgos de atributos. Los modelos pueden pertenecer a grupos politécticos: el grupo se define, no por un único atributo, sino sobre la base de un conjunto de atributos (los históricos) tales que cada entidad posee la mayor parte y cada uno de ellos es compartido por la mayoría de las entidades; son parecidos de familia (p. 55).

Esta definición resulta de utilidad para pensar el concepto de modelo institucional: ¿qué es lo que se comparte entre diferentes tipos de ofertas institucionales en términos de parecidos de familia? También es pertinente, para pensar lo que aquí se denomina sistematización estatal modelizadora, el siguiente interrogante: ¿qué se arrastra cuando una institución se define como parte de una red de formas similares-modelo en el sentido de parecidos de familia, atributos comunes en términos de curriculum y organización institucional?

Por su parte, Hillary Steedman (1992) crea el concepto de institución determinante al analizar el desarrollo de la escuela secundaria en Inglaterra. Este último refiere a aquellas instituciones que actuaron como autoridad en el campo de la enseñanza secundaria y que, por lo tanto, se convirtieron en el marco de referencia de las instituciones de educación secundaria que intentaban acercarse a ellas. Destaca la autora el valor puramente simbólico de este acercamiento ya que estas instituciones no guardaban relación con procesos efectivos de alteración de la reproducción social. Sin embargo, en el caso inglés, el conjunto del sistema de secundaria se organizó a imagen y semejanza de un tipo de institución: las public schools. ¿Es posible sostener esta función y modo de operar para el caso del colegio nacional en la Argentina?

La forma en que Burke define modelo permite pensar en la dinámica de la sistematización estatal modelizadora: aquello que se va transmitiendo entre ofertas institucionales en términos de parecidos de familia. La noción de Steedman habilita a analizar el lugar

1 Tomar como referencia el concepto de sistematización, que caracterizamos aquí como de sistematización estatal, implica la adopción de una perspectiva de análisis centrada en la acción del Estado nacional. No constituyen objeto de estudio de este trabajo perspectivas dedicadas a otro tipo de actores como, por ejemplo, estados provinciales o actores institucionales, las que seguramente aportarían a una comprensión más compleja. 
que el colegio nacional ocupó en la configuración de la oferta de educación secundaria, también desde una lógica de modelización.

Ambos conceptos componen la noción de modelo institucional. Este refiere a una forma organizacional -gramática de la escolarización-, más una forma pedagógica -la localización de esa gramática que confluye en un determinado régimen académico-, más la institucionalización de la escuela secundaria en términos de instituciones determinantes como modelos de referencia. A ello se suma una historia colectiva del establecimiento escuela secundaria en el marco de procesos de sistematización educativa.

El desarrollo del presente trabajo se organiza en tres apartados: el primero destaca hitos en los inicios de la sistematización estatal modelizadora; el segundo desarrolla el momento de perfeccionamiento de la sistematización estatal modelizadora; y el tercero analiza los efecto de dicha forma de la sistematización sobre la segmentación del sistema educativo. Utiliza fuentes primarias: decretos, planes de estudio y reglamentos escolares; $y$ fuentes secundarias en tanto estudios previos sobre el tema.

\section{Los inicios de la sistematización estatal modelizadora}

El Decreto 5447 para la creación del Colegio Nacional de Buenos Aires, en marzo de 1863, inauguró no sólo un establecimiento educativo sino más bien un modelo institucional que constituiría el modelo de los colegios nacionales durante los siguientes treintas años a partir de su fundación (Legarralde, 1997). Dicho decreto definió el colegio nacional como una casa de educación científica preparatoria en la que se cursarían las Letras, las Humanidades, las Ciencias Morales, Físicas y Matemáticas.

A este decreto se sumó, en diciembre de 1864, los de creación de los colegios nacionales de Catamarca, Tucumán, Mendoza, San Juan y Salta. Estos decretos establecían, en su artículo segundo: “...la enseñanza durará cinco años y se ajustará al Programa de estudios que rige en el Colegio Nacional de Buenos Aires que servirá de base, así como su reglamento para el régimen interno, estableciéndose además una clase especial de minerología" (García Merou, 1900: 130). El artículo cuarto agregaba la educación en dichos colegios de 20 niños pobres a cuenta de la nación. El resto de los artículos, referidos a la organización, la validez del certificado para acceder a las universidades nacionales y los requisitos de ingreso a los colegios, se mantuvieron iguales al decreto del colegio inaugural.

La existencia de estos decretos no permite suponer, en la práctica, la estandarización del modelo de origen, el de Buenos Aires, y el del resto de los colegios nacionales en creación. A pesar de ello, se sostiene aquí que esta voluntad estatal inicial de modelización instala una dinámica en el proceso de configuración y expansión, o de formación y perfeccionamiento, del modelo institucional. Ejemplos de ella se encuentran entre los colegios nacionales pero también entre otras ofertas de enseñanza. En el primer caso puede mencionarse, además de la creación de colegios en las provincias, la creación de secciones del Colegio Nacional de Buenos Aires en otros sectores de la ciudad.

En 1898, a raíz de que el Colegio Nacional de la Capital estaba excedido en su capacidad edilicia por una matrícula que crecía continuamente, habilitó cuatro secciones que luego se transformaron en colegios nacionales: las secciones Norte (luego Colegio Sarmiento), Sud (luego Colegio Rivadavia), Oeste (luego Mariano Moreno) y Noroeste (luego Colegio Nicolás Avellaneda); a cargo de los respectivos Vicerrectores que dependían del Colegio Buenos Aires que pasó a denominarse Central. 
En el segundo caso, en el Proyecto de Reformas a los Planes de Estudio de la Enseñanza Media (1934), se describe el proceso de creación de la enseñanza comercial e industrial. Según su autor, el entonces Inspector General de Enseñanza Secundaria, Normal y Especial, Juan Mantovani, durante la Presidencia de Pellegrini, se fundó la primera Escuela de Comercio Nacional en la Capital de la República, lo que define como el punto de partida del vigoroso impulso que recibe desde entonces la enseñanza comercial: la refundación de la Escuela de Comercio de la ciudad de Rosario en 1895, la creación de las escuelas de comercio en Concordia y Bahía Blanca en 1903. Sobre la modelización, destaca que en 1905, considerando insuficiente la capacidad de la Escuela de Comercio de la Capital Federal -posteriormente denominada Carlos Pellegrini- se creó la Sección Sur dependiente de aquella, que se independiza para constituir otra escuela comercial en 1909. Se fundó además la Escuela de Comercio de Tucumán en 1909 y la de La Plata en 1910. En cuanto a la enseñanza industrial, el mismo informe destaca que el ministro Magnasco separó el departamento industrial de la escuela de comercio y así fundó la Escuela Industrial de la Nación, Otto Krause.

La práctica de crear secciones de una misma institución no fue sólo propia del Colegio Nacional sino también de las primeras escuelas nacionales, comerciales e industriales. Asimismo, por la regulación impuesta a los colegios particulares -como se verá en el párrafo siguiente-, la incorporación de escuelas industriales a las nacionales también suponía la modelización conforme un plan de estudio de referencia.

En efecto, un segundo hito hacia la convergencia con el modelo institucional del Colegio Nacional es el de su consolidación como modelo para los establecimientos particulares. Desde la caída del gobierno de Juan Manuel de Rosas, en 1852, hasta 1878, los estudiantes de establecimientos particulares rendían sus exámenes en forma directa en la universidad; a partir de entonces, durante la presidencia de Avellaneda, los estudiantes particulares adquirieron el derecho de rendir los exámenes en los colegios nacionales. Para ello, el establecimiento particular debía incorporarse a un colegio nacional.

Los exámenes eran con un jurado mixto con mayoría de profesores del colegio nacional, donde se rendía el examen, y como presidente del jurado el rector o profesor del colegio nacional de más antigüedad (según Abeille, 1910, por uso y costumbre). Los estudiantes que aprobaban todos los exámenes obtenían un certificado de estudios emitido por el colegio nacional donde rendían. Estos certificados, mencionando la proveniencia del candidato, eran aceptados en las universidades.

El 1 de marzo de 1886 -bajo la presidencia de Roca-, el decreto reglamentario de la Ley sobre libertad de enseñanza establecía en su artículo 2, inciso 3, que era necesaria la designación de los años de estudios durante los que se iba a enseñar en el colegio particular según el plan de estudios vigente en el colegio nacional. Además, el artículo 3 establecía que, si los alumnos debían dar exámenes parciales, se manifestaría la aceptación del plan de estudios de los colegios nacionales y la preparación de aquellos de acuerdo con la distribución de las asignaturas establecidas en dicho plan. En el caso de dar exámenes generales, se debía manifestar la aceptación del plan de estudios de los colegios nacionales o acompañar el plan de estudios que rijiera en el colegio particular, el que comprendería, por lo menos, las mismas materias del plan oficial. El inspector de colegios nacionales tenía a su cargo el control de estas condiciones (García Merou, 1900).

De acuerdo con Abeille (1910), en su relato sobre la experiencia del Colegio Nacional de Buenos Aires, en 1910 este tenía incorporado al Colegio del Salvador (con 342 alumnos en 1906) y al Colegio Lacordaire, de la orden de los Franciscanos (con 119 alumnos en ese año). Según dicho autor, los estudiantes tenían la posibilidad de pasarse al colegio nacional, "cosa que ocurre muy a menudo al menos en los años superiores" (1910: 260). 
Es claro que la existencia de una ley y su reglamento no dice mucho sobre las prácticas: los planes de estudio y sobre todo las formas de enseñanza seguramente variaron entre colegios nacionales y colegios particulares. Además, conforme el avance del siglo $\mathrm{XX}$, el sector particular lograría tomar distancia de la regulación de origen mediante distintos pasos bien sintetizados por Schoo (2012: 100-101): recursos públicos para el pago de los salarios docentes (1947), organismo propio de supervisión educativa (1958), regulación del régimen de calificaciones, exámenes y promociones (1960) y desarrollo de planes de estudio diferentes a los oficiales aprobados por la autoridad oficial competente (1964).

Interesa aquí considerar la función de esta reglamentación en la consolidación del colegio nacional como modelo. Siguiendo la noción de modelo de Burke (2007), el colegio nacional posee la mayor parte de atributos por ser el de origen; si cada colegio particular debe seguir el plan de estudio y rendir examen para ser incorporado, comparte uno de los atributos esenciales y tiene un gran parecido de familia. Asimismo, esta exigencia hacia los colegios particulares no solo consolidaba al colegio nacional como modelo hacia otros sino también su propio modelo.

\section{El perfeccionamiento de la sistematización estatal modelizadora}

Un tercer hito en la perspectiva de una sistematización estatal modelizadora se puede localizar en la creación de ciclos comunes entre las distintas ofertas de enseñanza. Constituyen antecedentes en esta dirección los estudios de Schoo (2012) e Iglesias (2017). Las propuestas de cambio de los planes de estudio de los colegios nacionales fueron continuas entre 1870 y 1940 . Un momento de estabilización se encontró a partir de 1917 con la restauración del plan del ministro J. M. Garro de 1912 (Decreto 12 de febrero). Dicho plan proponía una enseñanza secundaria (colegios nacionales y liceos de señoritas) con finalidad integral y organizada en dos ciclos: inferior de cuatro años de formación general y superior de dos años. El plan solamente se aplicó hasta el cuarto año y se desvirtuó (Proyecto de Reformas a los Planes de Estudio de la Enseñanza Media, 1934), pero instaló la idea de ciclo, que luego fue retomada por el proyecto de 1934 y el de Ley de Instrucción Pública de 1939.

El éxito de la idea de ciclo en la enseñanza secundaria tuvo lugar a partir de 1942 con la puesta en marcha de los Decretos $\mathrm{N}^{\circ} 88584 / 41$ y No 107.107/41 bajo la gestión del ministro Guillermo Rothe. El primero de estos decretos aludía a la reforma del régimen de exámenes y promociones y la consecuente reforma de los planes de estudios. El cambio principal se vinculaba con el problema de la práctica del examen por asignatura al finalizar el curso, cuestión que había estado sujeta a cambios desde los años '20. El Reglamento de Clasificaciones, Exámenes y Promociones de 1942 (1943: 12) formalizó la propuesta del decreto: un examen de capacidad al terminar cada materia o grupo de materias al fin de cada ciclo. Dichos exámenes serían orales a excepción de Castellano y Literatura, con examen oral y escrito, materias de dibujo, caligrafía y trabajos prácticos.

El segundo aprobaba precisamente los nuevos planes de estudio para todas las ofertas de enseñanza media, ya formalmente designada de esa manera, incluyendo la división en dos ciclos y la creación de un ciclo común para el bachillerato y magisterio (Ministerio de Justicia e Instrucción Pública, 1941: 4). Los motivos para la creación del ciclo común estaban dirigidos a uniformar la formación básica con el fin de posponer la orientación hacia uno u otro tipo de estudio. Junto con ello, se buscaba limitar la "superproducción de maestros" (sic) incorporando, además, un examen selectivo para el ingreso al ciclo superior de magisterio. Entre otros cambios citados, cabe también destacar la unificación de los estudios de bachillerato para varones y mujeres mediante 
la supresión de los cursos de labores de los liceos de señoritas por su escaso aporte a la preparación para los estudios universitarios y la redundancia con el aprendizaje hecho durante la primaria en esa área.

No interesa tanto aquí ahondar en los detalles del llamado Plan Rothe sino en su función como herramienta de modelización. La tabla siguiente muestra la inclinación hacia la oferta curricular de los colegios nacionales para el futuro ciclo común:

Tabla 1. Cantidad de Horas semanales por asignatura para los tres primeros años de Colegios Nacionales y Escuelas Normales. 1941*

\begin{tabular}{|c|c|c|c|c|}
\hline Asignaturas & $\begin{array}{c}\text { Colegios } \\
\text { Nacionales } \\
1941\end{array}$ & $\begin{array}{c}\text { Escuelas } \\
\text { Normales } \\
1941\end{array}$ & $\begin{array}{l}\text { Plan Rothe } \\
\text { propuesta }\end{array}$ & $\begin{array}{l}\text { Plan Rothe } \\
\text { aprobado }\end{array}$ \\
\hline Castellano & 12 & 12 & 12 & 12 \\
\hline Matemática & 13 & 12 & 15 & 15 \\
\hline Historia & 12 & 9 & 12 & 12 \\
\hline Geografía & 9 & 7 & 9 & 9 \\
\hline Cs. Biológicas & 6 & 6 & 9 & 9 \\
\hline Física & - & 2 & - & - \\
\hline Química & - & 2 & - & - \\
\hline Música & 5 & 5 & 5 & 5 \\
\hline Francés & 10 & 8 & - & $\begin{array}{c}\text { Opción Francés } \\
\text { o Inglés } 12\end{array}$ \\
\hline Inglés & 8 & - & 12 & $\begin{array}{c}\text { Opción Francés } \\
\text { o Inglés } 12\end{array}$ \\
\hline Dibujo & 6 & 3 & 6 & 6 \\
\hline $\begin{array}{l}\text { Escritura y Dibujo } \\
\text { Lineal }\end{array}$ & - & - & 3 & 4 \\
\hline Educación Física & 6 & 6 & 6 & 6 \\
\hline
\end{tabular}

Nota: Elaboración propia con base en Ministerio de Justicia e Instrucción Pública (1941). Planes e estudios para Colegios Nacionales, Liceo de Señoritas, Escuelas Normales, Escuelas Nacionales de Comercio, y Escuelas Industriales. Informe de la Inspección General de Enseñanza y Decreto del Poder Ejecutivo. Buenos Aires: s/d.

*Se incluyen las asignaturas de formación general, no las específicas de la especialidad.

La propuesta del Plan Rothe consideró la tendencia en la distribución de horas del Colegio Nacional en los casos de Matemática, Historia, Geografía y Dibujo, y la mantuvo igual en los casos donde la distribución del colegio nacional y de la escuela normal era pareja, es decir, en Castellano, Música y Educación Física. En Ciencias Biológicas ambas ofertas tenían la misma distribución y el Plan Rothe pretendía aumentarla. Salvo en el caso de la opción por el Idioma (Francés o Inglés), el plan aprobado avanzó sobre la base de la propuesta realizada. La fusión completa del bachillerato y las escuelas normales se produjo en 1969: todas pasaron a ofrecer el título de Bachiller. ${ }^{2}$

En el caso de las escuelas comerciales, el Plan Rothe aconsejaba el mantenimiento de los tres ciclos que integraban el plan vigente desde 1939: el primero de tres años (para los cursos diurnos con título Auxiliar de Comercio) o de cuatro (para los nocturnos con 
título Tenedor de libros); el segundo de un año y el tercero también de uno (Título Perito Mercantil). Una mirada a la propuesta y a lo efectivamente aprobado para el caso del ciclo de tres años no evidencia la misma tendencia arriba descripta, es decir, la inclinación hacia una distribución de horas más cercana a la propia de los colegios nacionales.

Tabla 2. Cantidad de Horas semanales por asignatura para los tres primeros años de Colegios Nacionales, Ciclo Común Plan Rothe y Primer ciclo Escuelas Comerciales. 1941*

\begin{tabular}{|c|c|c|c|c|c|}
\hline Asignaturas & $\begin{array}{c}\text { Colegios } \\
\text { Nacionales } \\
1941\end{array}$ & $\begin{array}{c}\text { Plan Rothe } \\
\text { ciclo } \\
\text { común }\end{array}$ & $\begin{array}{l}\text { Escuelas } \\
\text { Comercio }\end{array}$ & $\begin{array}{c}\text { Plan Rothe } \\
\text { propuesta } \\
\text { Comercio }\end{array}$ & $\begin{array}{c}\text { Plan Rothe } \\
\text { aprobado } \\
\text { Comercio }\end{array}$ \\
\hline Castellano & 12 & 12 & 12 & 12 & 12 \\
\hline Matemática & 13 & 15 & 15 & 18 & 18 \\
\hline Historia & 12 & 12 & 9 & 6 & 6 \\
\hline Geografía & 9 & 9 & 9 & 9 & 9 \\
\hline Cs. Bio & 6 & 9 & - & - & - \\
\hline Inst. Cívica & - & - & 2 & 2 & 2 \\
\hline Física & - & - & 3 & 6 & Físico-Química 4 \\
\hline Química & - & - & 2 & 6 & Físico-Química 2 \\
\hline Música & 5 & 5 & - & Canto 2 & Canto 2 \\
\hline Francés & 10 & $\begin{array}{c}\text { Francés/ } \\
\text { Ing. } 12\end{array}$ & $\begin{array}{c}\text { Francés o } \\
\text { Inglés } 13\end{array}$ & 9 & Francés o Inglés 9 \\
\hline Inglés & 8 & $\begin{array}{c}\text { Francés/ } \\
\text { Ing. } 12\end{array}$ & $\begin{array}{l}\text { Francés o } \\
\text { Inglés } 13\end{array}$ & 9 & Francés o Inglés 9 \\
\hline Dibujo & 6 & 6 & - & - & - \\
\hline $\begin{array}{l}\text { Escritura y } \\
\text { Dibujo Lin }\end{array}$ & - & 3 & - & & \\
\hline $\begin{array}{l}\text { Caligrafía y } \\
\text { Dibujo Lin }\end{array}$ & - & - & Caligrafía 5 & 5 & 5 \\
\hline Ed. Física & 6 & 6 & 6 & - & 6 \\
\hline
\end{tabular}

Nota: Elaboración propia con base en Ministerio de Justicia e Instrucción Pública (1941). Planes e estudios para Colegios Nacionales, Liceo de Señoritas, Escuelas Normales, Escuelas Nacionales de Comercio, y Escuelas Industriales. Informe de la Inspección General de Enseñanza y Decreto del Poder Ejecutivo. Buenos Aires: s/d.

*Se incluyen las asignaturas de formación general, no las específicas de la especialidad

De todas maneras, sobre un total de 12 asignaturas (11 sólo en el primer año), solamente 3 correspondían a la especialidad: Contabilidad (con 11 horas semanales), Mecanografía y Estenografía. El resto, salvo Dibujo en los Bachilleratos e Instrucción Cívica y Ciencias Físico-Químicas en el ciclo comercial, eran las mismas asignaturas. El total de horas semanales era levemente mayor para la oferta comercial, 32 sobre 30 para el ciclo común de bachilleratos y normales. La distribución de horas era pareja en Castellano; la diferencia más notoria se registraba en Historia: junto con las tres asignaturas específicas, aquí es donde se marca todavía la distancia (o la jerarquía cultural).

La inclusión de la enseñanza comercial en el ciclo común de la enseñanza secundaria y normal se produjo en 1952, mediante el Decreto 5981, de aplicación ese mismo año a través del Decreto 11539. Este plan se aplicó para los cursos diurnos de las escuelas nacionales de comercio.

Por intermedio de la unificación se procuró facilitar a los alumnos el pase de una orientación a otra sin perder años en su carrera y evitar la superposición con asignaturas 
del ciclo superior. La tabla siguiente da cuenta de un proceso de generalización del currículum en sentido amplio.

Tabla 3. Cantidad de Horas semanales por asignatura para Ciclo Común y primer ciclo Escuelas comerciales Plan Rothe, Ciclo básico Enseñanza Secundaria 1952 y Ciclo Básico Escuelas de comercio diurnas 1952*

\begin{tabular}{|c|c|c|c|c|}
\hline Asignaturas & $\begin{array}{l}\text { Ciclo Común } \\
\text { Rothe }\end{array}$ & $\begin{array}{c}\text { Ciclo Básico ES } \\
1952^{* *}\end{array}$ & $\begin{array}{c}\text { Primer Ciclo } \\
\text { Comercio Plan } \\
\text { Rothe }\end{array}$ & $\begin{array}{c}\text { Escuelas comercio } \\
\text { diurnas } 1952\end{array}$ \\
\hline Castellano & 12 & 14 & 12 & 11 \\
\hline Matemática & 15 & 12 & 18 & 18 \\
\hline Historia & 12 & 9 & 6 & 9 \\
\hline Geografía & 9 & 9 & 9 & 9 \\
\hline Cs. Bio & 9 & 6 & - & 6 \\
\hline Inst. Cívica & - & $\begin{array}{c}\text { Cultura } \\
\text { ciudadana } 6\end{array}$ & 2 & $\begin{array}{c}\text { Cultura Ciudadana } \\
6 \\
\end{array}$ \\
\hline Física & - & Físico Química 2 & Físico-Química 4 & Físico-Química 2 \\
\hline Química & - & - & Físico-Química 2 & \\
\hline Música & 5 & Cultura Musical 5 & Canto 2 & Cultura Musical 2 \\
\hline Idioma & Francés o & F/l/opción & Francés o Inglés & F/l/opción \\
\hline extranjero & Ing. 12 & 9 & 9 & 9 \\
\hline Dibujo & 6 & 5 & - & - \\
\hline $\begin{array}{l}\text { Escritura y } \\
\text { Dibujo Lineal }\end{array}$ & 3 & - & - & - \\
\hline $\begin{array}{l}\text { Caligrafía y } \\
\text { Dibujo Lineal }\end{array}$ & - & - & 5 & $\begin{array}{c}\text { Ornamental } \\
5\end{array}$ \\
\hline Ed. Física & 6 & - & 6 & - \\
\hline Act. Prácticas & - & 2 & - & - \\
\hline $\begin{array}{l}\text { Act. Prácticas } \\
\text { (Contabilidad) }\end{array}$ & - & 2 & & $\begin{array}{c}\text { Contabilidad } \\
{ }_{11}\end{array}$ \\
\hline
\end{tabular}

Nota: Elaboración propia con base en Ministerio de Educación de la Nación. Dirección General de Enseñanza Secundaria, Normal, Especial y Superior (1953). Planes y programas de estudio. Ciclo Básico. Segundo Ciclo del Bachillerato. Ciclo Superior del Magisterio. Escuelas Normales Regionales. Escuelas Nacionales de Comercio. Buenos Aires: s/d.

* Se incluyen las asignaturas de formación general o aquellas que se generalizan, no las específicas de la especialidad como Mecanografía

**El plan de 1952 agrupaba las disciplinas por áreas de Formación (lingüístico-literaria, científica, histórico-social y de la conciencia nacional, religiosa, estético y práctica).

Al incorporarse al ciclo común, el plan comercial se apegó a la tendencia generalista: más horas de Historia, inclusión de Ciencias Biológicas, equiparación de las horas de Físico-Química con las otras ofertas. La diferencia son las horas de Contabilidad y la presencia de Mecanografía y Dibujo Ornamental. En menor medida, los bachilleratos y normales también profundizaron la tendencia generalista al incorporar Físico-Química y Contabilidad práctica. Este plan fue modificado en 1956 (Decreto 6680 del 13 de abril), en relación con la eliminación de la formación religión implantada durante el Peronismo. Hacia fines de los años ' 80 los cursos nocturnos se incorporaron al ciclo básico común.

Las escuelas industriales probablemente ameriten un tratamiento aparte dada la particularidad de su recorrido a partir de 1945 con la irrupción del Peronismo y la separación de la oferta, luego denominada técnica, entre la Comisión Nacional de Aprendizaje y Orientación Profesional (CNAOP) y la Dirección General de Enseñanza Técnica 
(DGET). La primera tuvo a su cargo las Escuelas Fábricas, que eran parte del primer ciclo de la CNAOP y tenían tres años de duración; funcionaban cumpliendo un plan mixto de enseñanza y producción durante 48 horas semanales y contaban con una planta industrial dedicada a la especialidad. Como condición de ingreso, se exigía escolaridad primaria, que podía suplirse excepcionalmente por examen equivalente.

El gobierno de la Revolución Libertadora del año 1955 fusionó dichos organismos en 1959, con la creación del Consejo Nacional de Enseñanza Técnica (CONET) con el objetivo de homogeneizar la formación técnica y profesional. Bajo la dirección del CONET quedaron entonces todas las escuelas técnicas, que fueron agrupadas bajo la denominación de Escuela Nacional de Educación Técnica (ENET).

Como destaca Díaz (2009), el currîculum de estas escuelas estuvo organizado conforme un ciclo básico (como las demás escuelas secundarias) de tres años y dos trayectos diferenciados por título. Los contenidos del curriculum se articularon desde una perspectiva tradicional, donde los saberes prácticos se subordinaban a los cientificos y luego de la teoría vendrîan las acciones prácticas desarrolladas en los talleres.

Más allá de esta dispersión de origen, es posible destacar algunos rasgos de la dinámica de modelización. Más arriba se señaló que la creación de las escuelas industriales siguió el patrón de creación de establecimientos a partir de desprendimientos de la institución de origen, en este caso la Escuela Industrial Nacional Otto Krause.

A su vez, el Plan Rothe estabilizó la oferta de ese tipo de instituciones mediante la consolidación de un primer ciclo común para todas las especialidades industriales de los colegios diurnos. Analizar la cantidad de materias y la distribución horaria de las asignaturas de formación general permite identificar algunos indicios hacia la generalización: más horas para Historia, Geografía y Biología y menos de Estética gráfica y Taller (ver Tabla 4).

Tabla 4. Cantidad de Horas semanales por asignatura para ciclo común Plan Rothe, Primer ciclo escuelas industriales 1941 y Plan Rothe

\begin{tabular}{|c|c|c|c|c|}
\hline Asignaturas & $\begin{array}{l}\text { Plan Rothe } \\
\text { ciclo común }\end{array}$ & $\begin{array}{c}\text { Primer ciclo } \\
\text { El } 1941\end{array}$ & $\begin{array}{l}\text { Plan Rothe } \\
\text { propuesta }\end{array}$ & Plan Rothe aprobado \\
\hline Castellano & 12 & $\begin{array}{l}\text { Idioma } \\
\text { Nac.10 }\end{array}$ & 12 & Castellano 12 \\
\hline Matemática & 15 & 18 & 18 & 18 \\
\hline Historia & 12 & - & 6 & 6 \\
\hline Geografía & 9 & - & 6 & 6 \\
\hline $\begin{array}{l}\text { Historia y } \\
\text { Geografía }\end{array}$ & - & 6 & - & - \\
\hline Cs. Biológicas & 9 & 4 & 6 & 6 \\
\hline Física & - & 7 & 6 & 6 \\
\hline Química & - & 3 & 3 & 3 \\
\hline Música & 5 & - & - & - \\
\hline $\begin{array}{l}\text { Idioma } \\
\text { extranjero }\end{array}$ & $\begin{array}{l}\text { Francés/ } \\
\text { Ing. } 12\end{array}$ & - & - & - \\
\hline Dibujo & 6 & - & - & - \\
\hline $\begin{array}{l}\text { Escritura y } \\
\text { Dibujo Lineal }\end{array}$ & 3 & 6 & 9 & 9 \\
\hline Dibujo a punto & - & 8 & 6 & 6 \\
\hline
\end{tabular}




\begin{tabular}{lcccc}
\hline \multicolumn{1}{c}{ Asignaturas } & $\begin{array}{c}\text { Plan Rothe } \\
\text { ciclo común }\end{array}$ & $\begin{array}{c}\text { Primer ciclo } \\
\text { El 1941 }\end{array}$ & $\begin{array}{c}\text { Plan Rothe } \\
\text { propuesta }\end{array}$ & Plan Rothe aprobado \\
\hline $\begin{array}{l}\text { Dibujo de } \\
\text { proyecciones }\end{array}$ & - & 3 & - & 3 \\
\hline Estética gráfica & - & 4 & 3 & 6 \\
\hline $\begin{array}{l}\text { Educación } \\
\text { Física }\end{array}$ & 6 & 6 & 6 & 33 \\
\hline Taller & - & 36 & 33 & \\
\hline
\end{tabular}

Nota: Elaboración propia con base en Ministerio de Justicia e Instrucción Pública (1941). Planes e estudios para Colegios Nacionales, Liceo de Señoritas, Escuelas Normales, Escuelas Nacionales de Comercio, y Escuelas Industriales. Informe de la Inspección General de Enseñanza y Decreto del Poder Ejecutivo. Buenos Aires: s/d.

A principios de 1960, se decidió aplicar en todas las escuelas de formación de técnicos los planes y programas que se venían usando en las Escuelas Industriales. Fue necesario adoptar un régimen transitorio para que todas se fueran adecuando, al cabo de unos años, para fundirse en un solo plan. Este proceso estuvo acompañado por una planificación de la oferta, especialmente en localidades donde se habían creado escuelas dependientes de ambos organismos, desarrollados durante el Peronismo, con fines y objetivos similares y donde no se justificaba más de un establecimiento (Planes 1956-1967).

La acción del ministro Rothe se extendió al modelo institucional más allá del cambio curricular: en efecto, encomendó la realización del Reglamento General para los Establecimientos de Enseñanza Secundaria, Normal y Especial, aprobado en 1943 (Schoo, 2015). En 1957, se volvieron a reordenar bajo un mismo reglamento todas las modalidades, sobre la base del reglamento de 1943, incorporando y reordenando el conjunto de normas que se fueron aprobando entre ambas fechas. Por ello, se aclaraba que no se trataba de un nuevo Reglamento General, "ni siquiera de la reforma del existente" (página XII).

El Reglamento General para los Establecimientos de enseñanza secundaria, normal y especial, aprobado en 1957, estuvo en vigencia hasta fines de la década de 1980 más allá de que existieran leyes, decretos y resoluciones ministeriales posteriores que fueron ampliando o restringiendo el sentido de sus artículos. Una vez más, en 1965 existió un reglamento específico para las ofertas técnicas. Las normas y prácticas, de todas maneras, tendieron a dispersarse conforme aumentó el peso de la oferta provincial entre los años '70 y ‘ 80 .

\section{Una posible síntesis: sistematización modelizadora y segmentación por diferenciación institucional}

Hasta aquí se delinearon los modos en que el Estado nacional, mediante la creación y modificación de distintos tipos de oferta destinada a la segunda enseñanza, llevó a cabo un proceso sistematización sobre la base de una tendencia modelizadora. En un comienzo lo hizo a través del modelo institucional del colegio nacional como referencia para creaciones provinciales y para escuelas particulares; y luego, por medio de la unificación de las distintas ofertas en ciclos comunes que siguieron un proceso de generalización creciente en sus asignaturas. Todas las ofertas participaron, en mayor o menor medida y con ritmos diferentes, de este proceso, el que se vio favorecido a su vez por la propia práctica de modelización entre las primeras escuelas comerciales $y$, sobre todo, entre las industriales. 
¿De qué manera se vincula la sistematización estatal modelizadora con la segmentación del sistema educativo? Entre fines del siglo XIX y durante las primeras décadas del siglo $\mathrm{XX}$, se configuraron las principales formas institucionales de la segunda enseñanza. $\mathrm{Al}$ respecto, en el Proyecto de Reformas a los Planes de Estudio de la Enseñanza Media (1934), el entonces Inspector General de Enseñanza Secundaria, Normal y Especial, Juan Mantovani, formulaba:

La enseñanza media viene a ser el género. Enseñanza secundaria, enseñanza normal, enseñanza comercial y enseñanza industrial serían las especies. Al hablar en este informe de enseñanza media se hace referencia a todas las ramas anotadas, mientras que cuando se dice enseñanza secundaria la designación alcanza, única y estrictamente, a la que se imparte en los colegios nacionales y liceos de señoritas, o sea, a los estudios de bachillerato.

En nuestro país, siempre que se pensó en la reforma de la enseñanza media, se consideró únicamente la reforma del colegio nacional. Los demás institutos de enseñanza media, fundados para responder a exigencias locales, circunstanciales o particulares, nunca tuvieron mayor relación con el conjunto del sistema de esa enseñanza. Hasta la misma denominación de enseñanza media es más bien un nombre sin contenido determinado, pues en nuestro país no ha habido una enseñanza media, sino ramas inconexas, cada cual con su particular orientación, cuando la tenía.

En las reformas que se proponen, la etapa media contiene diversos tipos de establecimientos de enseñanza, de cuya doble finalidad -general, es decir, para todas las ramas, y específica de cada una- se tratará más adelante. Conviene, sin embargo, tener en cuenta las diversas especialidades que imparten, y sus correspondientes certificados o títulos de terminación de estudios: enseñanza secundaria (bachillerato), enseñanza normal (magisterio primario), enseñanza comercial (perito comercial), enseñanza industrial (técnica industrial en cuatro especialidades: mecánica, electricidad, construcciones y química, las que podrían aumentarse, conforme a nuevas exigencias). (1934: 113)

Las palabras de Mantovani dan cuenta de la configuración segmentada del sistema educativo argentino, con orientaciones inconexas luego de la escuela primaria. Esta forma que adquirió, en el caso argentino, el proceso de sistematización estatal y segmentación también tuvo sus particularidades. Algunos autores destacan a lo largo de este proceso una segmentación primero vertical, entre tipos de ofertas, y luego horizontal, con la creación de ciclos comunes y superiores y las distintas formas de acceso que suponían (Díaz, 2009; Iglesias, 2017; Schoo, 2012).

Se propone aquí que cabe advertir otra forma de segmentación, probablemente producto del intento constante por conducir las distintas ofertas hacia la formación general mediante la sistematización estatal modelizadora. Braslavsky (1985) la denominó diferenciación institucional y la registró hacia mediados de los años ' 80 :

El sistema educativo argentino está sin duda alguna fuertemente segmentado [...] el proceso de segmentación escolar se inició en realidad en el preescolar y se continuó a través de una serie de episodios [...] (49-57)

[...] El detonante dentro del sistema educativo son los mecanismos de ingreso que generan los órganos de gobierno y los establecimientos de nivel secundario. Pero la escuela primaria -y a esto se quiso apuntar- responde de manera funcional. (117)

[...] El acceso o no acceso de los adolescentes de los sectores populares urbanos al nivel secundario está fuertemente condicionado por el segmento del sistema de educación primaria del que se egresa. (120) 
[...] Por lo tanto el resultado del proceso de pasaje no es solo el acceso o no al nivel secundario sino el acceso diferenciado a sus distintos segmentos. (125)

[...] Estos datos invitan por lo menos tres reflexiones: 1) sobre la permanencia del valor social del colegio nacional para toda la población y en particular para sus sectores medios y altos, 2) sobre cómo se trazan las líneas de selección social por modalidades y 3) sobre la importancia de la institución escolar primaria sobre la orientación entre las modalidades del nivel secundario. (127-128)

Es posible rastrear marcas previas de diferenciación institucional a las detectadas por Braslavsky. Una de ellas se encuentra en la anexión de los colegios de Córdoba y La Plata a las respectivas universidades en 1907. Circuito que se completó con el decreto del 4 de noviembre de 1911, efectivizado recién en 1919, para la anexión del Colegio Nacional de Buenos Aires (Central) a la Universidad de Buenos Aires. Se sumaría luego la Escuela Nacional Superior de Comercio Carlos Pellegrini. El esquema se extendió a la Universidad del Litoral con la Escuela Industrial y Comercial de Rosario y tendió a repetirse conforme se crearon nuevas universidades nacionales.

Si bien las universidades debían atender los planes de estudio del Estado nacional, tenían la posibilidad de orientar la formación hacia la función preparatoria, frente a la función generalista que adquiría peso en la medida que aumentaba la oferta de bachilleratos. Esta marca se articuló con otra, efecto del Plan Rothe. Además de la creación de ciclos, este plan proponía un Bachillerato con latín para los ciclos básicos compartidos por universidades nacionales. La función preparatoria recuperó así la distinción específica de la jerarquía cultural: el latín.

Las reformas integrales no tuvieron cabida en la extensión de la enseñanza media, como ya se la denomina desde los años '40. No hubo reformas de tipo comprehensivo como las descriptas en el caso de Europa y Estados Unidos (Acosta, 2012). Según Ruiz (2012), la evolución histórica de la educación secundaria no estuvo acompañada por una política integral de organización institucional y académica. El Plan Rothe de 1941 fue la acción más orgánica que llevó adelante el Estado nacional para otorgarle propósitos y objetivos de formación a la enseñanza secundaria, hasta 1993.

Una excepción se produjo en 1969, en el marco de lo que se denominó la Reforma Educativa, ${ }^{3}$ con el ensayo de modificación de la escuela secundaria. Esta reforma creó una organización departamental que contaba, entre otros, con un Departamento de Orientación, integrado por las figuras de asesor pedagógico o psicopedagógico y un ayudante de departamento. Además, se redefinió al preceptor como un auxiliar docente. Estos cambios procuraban atender las diferencias individuales de los alumnos a través de tutoría, nivelación y orientación, así como favorecer la autonomía y crecimiento de los adolescentes mediante una reforma del régimen disciplinar. En el plano curricular, apuntaban a producir modificaciones tales como reducir el número de asignaturas diarias, reestructurar los programas en unidades didácticas correlacionadas, agrupar varias materias con un solo profesor, programar actividades curriculares y extracurriculares y actividades optativas u obligatorias los sábados, realizar actividades programadas en el contraturno. El Departamento de Orientación y su personal asesor tenían la doble función de complementar la tarea docente asistiendo en la atención individual de los alumnos y en las tareas de planificación curricular (Acosta y Pinkasz, 2014).

3 Durante el gobierno militar del general Onganía, se inició una ambiciosa reforma del Sistema Educativo. Esta reforma tuvo una fuerte oposición política y gremial y se llevó a cabo solo parcialmente. De la misma perduran algunos cambios como la formación de maestros en el nivel terciario y algunas escuelas con el régimen de designación de profesores por cargo (Proyecto 13), aunque con pocos de sus rasgos originales. 
Para concretarlos, se reformó el régimen de contratación de los profesores y es por esta razón que se lo denominó Régimen Laboral de Profesores Designados por Cargo Docente, aunque fue y es aún hoy conocido como Proyecto $13 .{ }^{4}$ El Proyecto 13 implicó la modificación de la estructura curricular en forma de damero -menos disciplinas y menos profesores-, procuró dar lugar al desempeño de nuevas funciones por parte del profesor - programación del currículum- e introdujo funciones de apoyo a su tarea por parte de profesionales especializados -asesoramiento, orientación y tutoría a alumnos. Además incorporó algunas de las novedades de la época: la idea de la programación del currículum por parte de los docentes e incorporó en los establecimientos a los nuevos profesionales de la educación como los de Ciencias de la Educación y de Psicopedagogía.

Sin embargo, la implementación del Proyecto 13 se limitó a las escuelas donde se lo aplicó experimentalmente, dados los costos que suponía trasladarlo a mayor escala. Autores como Braslavsky (1985) y Gallart (2006) destacan la preferencia de los padres por este tipo de escuelas entre mediados de los años ' 80 y ‘ 90 , profundizando el proceso de segmentación por diferenciación institucional arriba postulado.

Finalmente, otra marca que indica dicho proceso puede vincularse con la práctica de los exámenes de ingreso. En sus orígenes, los criterios de ingreso a los colegios nacionales estuvieron asociados a la acreditación de saberes básicos. Conforme aumentó la escolarización primaria, el certificado de estos estudios constituyó el principal mecanismo. En la década de 1940 se establecieron criterios comunes relacionados con los requisitos de ingreso a todas las ofertas: la edad de ingreso en los 12 años cumplidos y la toma de los exámenes de ingreso en las instituciones en las que la cantidad de inscriptos superara las vacantes, a excepción de aquellos ubicados en la Capital Federal. Los exámenes se redujeron a Castellano y Matemática para todas las modalidades.

En 1957 todo esto fue ratificado, pero agregando a las escuelas del Gran Buenos Aires en la obligación de tomar examen de selección, aunque las vacantes resultaran suficientes para la cantidad de inscriptos. Las escuelas más demandadas pudieron así seleccionar a través de un criterio meritocrático (Ministerio de Educación y Deportes, 2016).

Fue en la década de 1980, con la recuperación democrática, cuando se eliminaron definitivamente los exámenes de ingreso en los establecimientos nacionales de educación secundaria dependientes del Ministerio de Educación. La técnica del sorteo suponía una mayor democratización en las escuelas más demandadas. Sin embargo, las escuelas dependientes de las universidades nacionales mantuvieron la práctica de examen de ingreso. A su vez, el peso creciente de la jurisdicción provincial podría haber reforzado el mantenimiento de la diferenciación institucional por medio de la creación de nueva oferta institucional para descomprimir la demanda sobre las escuelas céntricas tradicionales.

\section{Conclusiones}

A lo largo del artículo se intentó mostrar de qué manera el modelo institucional de la escuela secundaria se formó sobre la base del modelo del Colegio Nacional, pero se perfeccionó en diálogo con las otras modalidades creadas como oferta de segunda enseñanza. 
El Colegio Nacional fue concebido como una institución modelo a la cual había que seguir e igualar y, en tanto institución determinante, se convirtió en el marco de referencia del conjunto de la educación secundaria. Esto también se expresó en el valor social otorgado a esta institución por sobre las demás, valor social expresado en la jerarquía cultural que representaba.

El trabajo partió de los aportes de los estudios histórico-comparativos y de la Historia de la Educación. Se eligieron conceptos que sirviesen al desarrollo teórico de la noción de modelo institucional en el marco de la configuración de los sistemas educativos. Sistematización y segmentación fueron considerados en sus aportes a la comprensión del lugar que dicha escuela ocupó en las formas de la expansión escolar. El concepto de modelo de Burke (1994) y el de institución determinante de Steedman (1992) permitieron, a su vez, comprender la función de una forma organizacional y pedagógica en términos de autoridad en el campo de la enseñanza secundaria y que, por lo tanto, se convirtieron en el marco de referencia de las instituciones de educación secundaria que intentaban acercarse a ellas.

El concepto de modelo institucional supone la condensación de tres elementos: por un lado, una cultura de la escolarización, que incluye las variables de la gramática de la escuela sumada a aquellas propias de la sistematización; por otro lado, la localización de esa cultura, que depende de cada escenario y confluye en distintas formas académicas; finalmente, la institucionalización de la escuela secundaria en tanto forma histórica, en términos de instituciones determinantes en el marco de procesos de sistematización educativa.

Desde nuestra perspectiva, la tendencia generalista constituyó la pieza clave. Hubo un modelo institucional determinante, pero su formación y perfeccionamiento durante la expansión fue producto de hibridaciones que buscaron al mismo tiempo proveer una oferta generalista y resguardar espacios de jerarquía cultural. Esto tuvo lugar en el marco de una sistematización estatal modelizadora y una segmentación centrada en la diferenciación institucional.

En efecto, hacia mediados de los años ' 80 del siglo XX el sistema educativo argentino se encontraba segmentado. La escuela secundaria, estructurada sobre la base de un modelo institucional centrado en el colegio nacional, cumplió la función de orientación de los públicos ingresantes. La tendencia modelizadora, sobre la base de la formación general podría haber favorecido procesos de diferenciación institucional particulares, centrados en dinámicas institucionales antes que sistémicas.

El estudio sobre el modelo institucional de origen de la escuela secundaria sirve para poner en discusión el alcance y las limitaciones de las políticas de expansión del sistema educativo que suelen realizarse sin analizar los modelos institucionales sobre los que se propone dicha expansión. Precisamente, los procesos de sistematización educativa llevaron a la escuela secundaria a una tensión entre expansión y modelo institucional, transformándola así en un problema a atender desde las políticas; los modos de resolver dicha tensión fueron las reformas de la escuela secundaria (Acosta, 2015).

Algunos países avanzaron más que otros en atender este problema. Todos los países, más allá de su grado de avance, presentan en la actualidad casos de políticas educativas dirigidos a atender esta problemática en un abanico que va desde las políticas orientadas hacia la transformación del modelo institucional, hasta aquellas ligadas a la acentuación de procesos de diferenciación institucional.

Más jóvenes en la escuela secundaria siempre es un dato positivo. Pero también implica un desafío: significa revisar las prácticas existentes porque muchas veces son las condiciones de la escolarización las que expulsan a los nuevos estudiantes. Esto no solo 
en lo referente a los dispositivos de los modelos institucionales, tal como proponen programas recientes, sino en cuanto a los procesos históricos de diferenciación institucional y sus efectos sobre la segmentación educativa. 


\section{Bibliografía}

" Acosta, F. (2012). La escuela secundaria argentina en perspectiva histórica y comparada: modelos institucionales y desgranamiento durante el siglo XX. Cadernos de História da Educaçao (11), pp. 31-144.

"Acosta, F. (2014). Entre procesos globales y usos locales: Análisis de categorías recientes de la Historia de la educación para el estudio de la escuela secundaria en la Argentina. Tiempo, Espacios, Educación, 1 (2), pp. 23-37.

» Acosta, F. (2015). La dinámica de incorporación y expulsión en la escuela secundaria: Modelos institucionales y desgranamiento en el ámbito nacional y local. Informe final de investigación Proyecto 30/3156. Universidad Nacional de General Sarmiento.

» Acosta, F. y Pinkasz, D. (2014). La escuela secundaria como objeto de estudio: lecturas desde la Historia de la educación. XVIII Jornadas Argentinas de Historia de la Educación. Universidad Nacional de General Sarmiento.

"Abeille, L. (1910). L'esprit démocratique de l'enseignement secondaire argentin 1810-26 mai-1910. París: Libraire Honoré Champion.

"Braslavsky, C. (1985). La discriminación educativa en la Argentina. Buenos Aires: FLACSO/Gel.

» Burke, P. (2007). Historia y teoría social. Buenos Aires: Amorrortu.

"Díaz, N. (2009). Procesos de estructuración y definición del currículum para el ciclo básico de la escuela secundaria en la transición democrática argentina (1983-1989). (Tesis inédita de maestría). Universidad de San Andrés.

"Dussel, I. (1997). Currículum, humanismo y democracia en la enseñanza media (18631920). Buenos Aires: Flacso/Oficina de Publicaciones del CBC, Universidad de Buenos Aires.

» Gallart, M. A. (2006). La construcción social de la escuela media: una aproximación institucional. Buenos Aires: Stella.

"Iglesias, A. (2017). Plan Rothe: la consolidación del ciclo básico para la escuela media argentina a través de los discursos e historias profesionales de la burocracia educativa (1941-1946). (Tesis inédita de maestría). Universidad Nacional de La Plata.

"Legarralde, M. (1998). La fundación de un modelo pedagógico: los colegios nacionales entre 1862 y 1887 . Propuesta Educativa (21), pp. 38-43.

» Ministerio de Educación y Deportes (2016). Sobre la selectividad de la educación secundaria en Argentina. Análisis histórico sobre el régimen de evaluación y promoción. Informe elaborado por Susana Schoo. Dirección Nacional de Investigación y Estadística Educativa. Serie de Investigación.

"Mueller, D. (1992). El proceso de sistematización: el caso de la educación secundaria en Alemania. En Mueller, D.; Ringer, F. y Simon, B. (Comp.), El desarrollo del sistema educativo moderno. Cambio estructural y reproducción social 1870-1920. Madrid: Ministerio de Trabajo y Seguridad, pp. 37-86.

"Ringer, F. (1979). Education and society in Modern Europe. Bloomington: Indiana University Press.

» Ruiz, G. (2012). La institucionalización de la educación en sistemas escolares: su estructura académica. En Ruiz, G. (Coord.), La estructura académica argentina: 
Análisis desde la perspectiva del Derecho a la educación. Buenos Aires: Eudeba, pp.1328.

"Schoo, S. (2012). La organización de la educación secundaria, normal y especial en la Argentina. En Ruiz, G. (Coord.), La estructura académica argentina: Análisis desde la perspectiva del Derecho a la educación. Buenos Aires: Eudeba, pp. 91-138.

"Schoo, S. (2015). Informe sobre Planes de estudio y Reglamentos. En Acosta, F. (2015). La dinámica de incorporación y expulsión en la escuela secundaria: Modelos institucionales y desgranamiento en el ámbito nacional y local. Informe final de investigación Proyecto 30/3156. Universidad Nacional de General Sarmiento, pp. 10-23.

"Steedman, H. (1992). Instituciones determinantes: las "endowed grammar schools" y la sistematización de la educación secundaria inglesa. En Mueller, D.; Ringer, F. y Simon, B. (Comps.), El desarrollo del sistema educativo moderno: cambio estructural y reproducción social 1870-1920. Madrid: Servicio de Publicaciones, pp.161-194.

"Viñao, A. (2002). Sistemas educativos, culturas escolares y reformas. Madrid: Morata.

\section{Fuentes primarias}

»Decreto № 88584 del 17 de abril de 1941, que encarga “a la Inspección General de enseñanza el proyecto de un régimen de exámenes y promociones y la reforma de los actuales programas de estudios, de conformidad con las bases que en el mismo se establecen". En Boletín del Ministerio de Justicia e Instrucción e Instrucción Pública (16), 1941, pp. 100-102. Recuperado de <http://www.bnm.me.gov.ar/giga1/ normas/13717.pdfs

"García Merou, J. (1900). Leyes, decretos y resoluciones sobre Instrucción Superior, secundaria, normal y especial. Años 1810 a 1880 (tomo I). Buenos Aires: Taller Tipográfico de la Penitenciaría Nacional.

"García Merou, J. (1900). Leyes, decretos y resoluciones sobre Instrucción Superior, secundaria, normal y especial. Años 1881 a 1900 (tomo II). Buenos Aires: Taller Tipográfico de la Penitenciaría Nacional.

» Ministerio de Justicia e Instrucción Pública (1941). Planes de estudios para Colegios Nacionales, Liceo de Señoritas, Escuelas Normales, Escuelas Nacionales de Comercio, y Escuelas Industriales. Informe de la Inspección General de Enseñanza y Decreto del Poder Ejecutivo. Buenos Aires: s/d. Recuperado de <http://www.bnm.me.gov.ar/ giga1/documentos/ELo01251.pdf>

»Reglamento General para los Establecimientos de Enseñanza (1943). Departamento de Instrucción Pública. Ministerio de Justicia e Instrucción Pública. Buenos Aires: Talleres Gráficos de la Penitenciaría Nacional.

\section{Felicitas Acosta}

Doctora en Ciencias Sociales, Facultad Latinoamericana de Ciencias Sociales (Argentina); Magister en Ciencias Sociales con Orientación en Educación, Facultad Latinoamericana de Ciencias Sociales (Argentina); Licenciada en Ciencias de la Educación, Universidad de Buenos Aires. Investigador docente Universidad Nacional de General Sarmiento. Profesora Universidad Nacional de La Plata. Argentina. facosta@campus.ungs.edu.ar 